\section{Periodic filters with a three-mirror Gire-Tournois resonator in a Michelson interferometer}

\author{
Li Wei \\ John W. Y. Lit* \\ Wilfrid Laurier University \\ Department of Physics and Computer \\ Science \\ Waterloo, Ontario, N2L 3C5 \\ Canada \\ E-mail: Iwei@wlu.ca
}

\begin{abstract}
A periodic optical filter based on a Michelson interferometer with a three-mirror Gire-Tournois resonator in one of the two arms is analyzed. We derive the general condition for the occurrence of flattop spectra. The results show that flattop spectra can be obtained by suitably choosing the reflective coefficients of individual mirrors. (c) 2005 Society of Photo-Optical Instrumentation Engineers. [DOI: 10.1117/1.1885489]
\end{abstract}

Subject terms: optical filters; Gire-Tournois resonators; dense wavelength-division multiplexing.

Paper L040228 received May 3, 2004; revised manuscript received Jan. 19, 2005; accepted for publication Feb. 4, 2005; appeared online Feb. 7, 2005; published online Apr. 7, 2005.

\section{Introduction}

One of the key components in DWDM systems is the multi-channel periodic optical filter. In order to avoid loss penalties, a filter with a flattop spectrum is highly desirable. Devices based on waveguides ${ }^{1}$ and optical fibers, including fiber delay lines ${ }^{2}$ and fiber Bragg gratings ${ }^{3}$ have been proposed to create flattop periodic filters. Recently, Dingle et al. ${ }^{4,5}$ proposed a robust approach by using a two-mirror Gire-Tournois resonator (2MGTR) in one of the interferometer arms to achieve flattop spectral response, while Hsieh et al. ${ }^{6}$ used two 2MGTRs, one in each interferometer arm. In this paper, we present a periodic flattop filter, based on a multi-cavity, three-mirror Gire-Tournois resonator (3MGTR), in a Michelson interferometer (MI). We present the analytical expression for the optimum conditions for the occurrence of flattop spectral response. By using a multicavity etalon, the filter has many sets of optimum values, greatly relaxing the design conditions needed to achieve superior device performance in terms of flattop bandwidth and steepness. We note that while optical filters may be analyzed by using a Z-transform in a signal processing approach, ${ }^{7}$ our approach is based on two-beam interferometry analysis with physical considerations to obtain analytical expressions for the optimum design.

\footnotetext{
*Also with University of Ontario Institute of Technology, Oshawa
} Ontario, L1H 7L7 Canada

0091-3286/2005/\$22.00 @ 2005 SPIE

\section{Filter Architecture and Optimal Design}

The periodic optical filter under study is shown in Fig. 1. It is a modified MI, consisting of one 50:50 beam splitter, one $100 \%$ reflective mirror $\left(\mathrm{M}_{3}\right)$, and a 3MGTR. The lengths of the arms of the interferometer are $L_{1}$ and $L_{2}$. The 3MGTR is a special two-cavity Fabry-Perot etalon with two partially reflective mirrors $\left(\mathrm{M}_{1}\right.$ and $\left.\mathrm{M}_{2}\right)$ and one $100 \%$ reflective mirror $\left(\mathrm{M}_{0}\right)$ at rear. The cavity length of the etalon is $d$, and the lengths of the two cavities are identical. The reflective coefficients of $\mathrm{M}_{1}$ and $\mathrm{M}_{2}$ are $r_{1}$ and $r_{2}$, respectively. To find the complex reflection coefficient of a 3MGTR, the 3MGTR can be considered as a $2 \mathrm{MGTR}$ constructed with a partially reflecting mirror $\mathrm{M}_{3}$ and an equivalent reflecting mirror $2 \mathrm{MGTR}$ with a relative phase shift of $\theta_{2 M}$, given in Refs. 8 and 4 . Thus, the reflection coefficient $r_{3 M}$ and the relative phase shift $\theta_{3 M}$ of a 3MGTR can be obtained by using the results of the 2MGTR:

$r_{3 M}=\frac{-r_{2}+e^{-i\left(2 \delta+\theta_{2 M}\right)}}{1-r_{2} e^{-i\left(2 \delta+\theta_{2 M}\right)}}=e^{i \theta_{3 M}}$,

$\theta_{3 M}=-2 \tan ^{-1}\left[a_{2} \tan \left(\delta+\theta_{2 M} / 2\right)\right]$,

with

$\theta_{2 M}=-2 \tan ^{-1}\left(a_{1} \tan \delta\right)$

$a_{1,2}=\left(1+r_{1,2}\right) /\left(1-r_{1,2}\right)$,

where $\delta=n \beta d$, is the one-way phase factor of the single cavity of 3MGTR; $a_{1}$ and $a_{2}$ are defined as reflective factors; $n$ is the refractive index of the material of the cavity; $\beta=2 \pi / \lambda$ is the propagation constant in free space with $\lambda$ the wavelength of light in vacuum. The 3MGTR is an allpass filter and the resultant amplitude of the electrical field is unity while the resultant phase is determined by the reflective coefficients of the mirrors. This is the property that gives the filter its specific characteristics.

In general, to ensure that the spectrum of the transmitted output will have a flattop response, the one-way phase factor $\delta$ has to be equal to twice the phase difference between the two interferometer arms, i.e., $\delta=2 \beta \Delta L,{ }^{4-6}$ here $\Delta L_{1}$ $-L_{2}$ is the difference of the lengths from the beam splitter to the two arms. Then the normalized output intensity can be written as

$\left\{1+\cos \left[\theta_{3 M}+\delta\right]\right\} / 2$.

In order to obtain a unity transmission, the following equation has to be satisfied:

$-2 \tan ^{-1}\left\{a_{2} \tan \left[\delta-\tan ^{-1}\left(a_{1} \tan \delta\right)\right]\right\}+\delta=2 m \pi$.

One can see that a unity transmission is always present when $\delta=2 m \pi$, which is the resonance condition of a 3MGTR and gives the position of the center peak. To further simplify Eq. (6) one can also find the second condition for unity transmission

$2\left(1-a_{1}\right) a_{2}=\left[1+a_{1} \tan ^{2} \delta\right]\left[1-\tan ^{2}(\delta / 2)\right]$. 


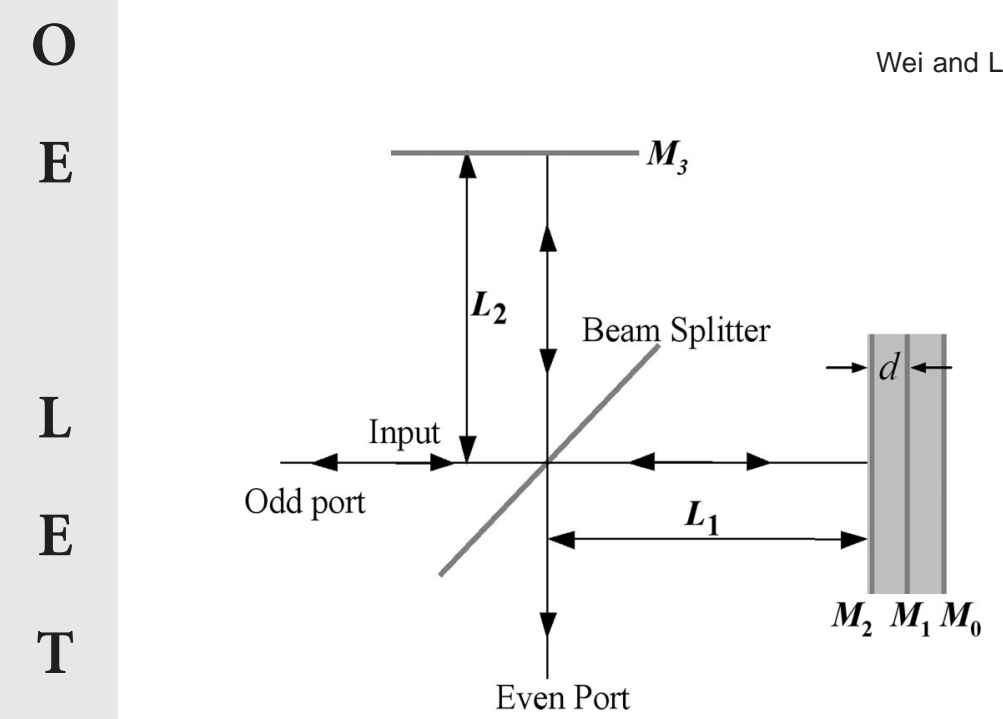

$\mathrm{T}$

E

$\mathbf{R}$

Fig. 1 Schematic of three-mirror Gire-Tournois resonator based Michelson interferometer.

which gives two side peaks. By substituting $\delta=2 m \pi$ into Eq. (6), Eq. (6) becomes:

$2 a_{2}\left(1-a_{1}\right)=1$.

With this condition, the triple peaks will overlap and give the transmission peak a flat top, and Eq. (8) is the optimum condition for flattop spectral responses. As an example, in Fig. 2(a) we plot the transmission curves with the reflective coefficient $r_{2}$ as a parameter and $r_{1}$ fixed at -0.68 . The optimum value of $r_{2}$ is -0.24 if we choose $r_{1}$ to be -0.68 according to Eq. (8). Figure 2(a) clearly shows that with the optimal design, the transmission curve has a flattop spectrum shown as a solid line. When the reflectivity of $\mathrm{M}_{2}$ is lower or higher than the optimum value, the spectrum has triple peaks shown as a dotted line, or a single peak with a rounded top shown as a dashed line.

Equation (8) tells us that there are numerous pairs of $r_{1}$ and $r_{2}$, which would give the flattop spectral responses for 3MGTR based filters. Of specific interest are the following two special cases when the 3MGTR becomes a 2MGTR. The first situation is when $r_{1}=-1$, i.e., $a_{1}=0$. The 3MGTR becomes a 2MGTR. By using Eq. (8), we can find that the general optimum condition for a 2MGTR-based periodic filter is $a_{2}=1 / 2$, which gives rise to $r_{2}$ $=-1 / 3$. Another extreme situation is when $r_{1}=-1 / 3$, i.e., $a_{1}=1 / 2$, which results in $r_{2}=0$. This again gives a 2MGTR-based periodic filter. Both results are consistent with the one shown in Ref. 5. The 3MGTR allows much more suitable choices for optimal designs compared with only one optimal value for the 2MGTR. More importantly, the 3MGTR-based design may enhance the optical performance of the filters in terms of the bandwidth of the flattop region and steepness, as can be seen in Fig. 2(b). Figure 2(b) gives the transmission spectra for three different configurations: standard MI, 2MGTR-based MI, and 3MGTR based MI. In Fig. 2(b), the reflective coefficients of the mirrors are chosen to be 1 for the standard MI, $-1 / 3$ for the partially reflective mirror of $2 \mathrm{MGTR}$, and -0.63 and

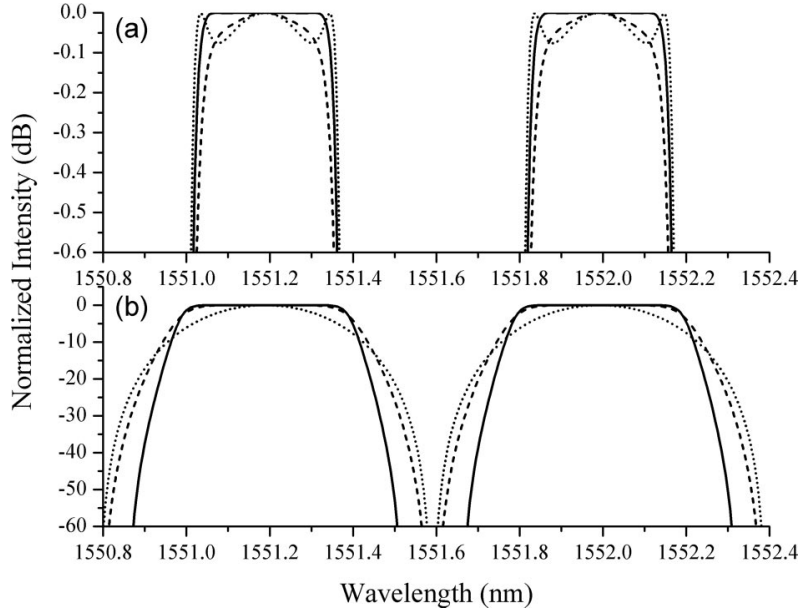

Fig. 2 (a) Normalized intensities of 3MGTR based MI with reflective coefficient $r_{2}$ of -0.14 (dotted line), -0.24 (solid line), and -0.34 (dashed line). (b) Normalized intensities for different configurations [MI (dotted line); 2MGTR based MI (dashed line); 3MGTR based MI (solid line)].

-0.214 for the two partially reflective mirrors of 3MGTR. Figure 2(b) clearly displays that the 3MGTR-based MI has an improved performance compared with the 2MGTRbased MI or the standard MI in terms of bandwidth and slope.

\section{Conclusions}

To summarize, we have theoretically studied periodic optical filters based on a multi-cavity GTR-based MI. The general condition for the occurrence of flattop transmission passband for a 3MGTR-based periodic filter is derived. The result shows that a 3 MGTR-based filter is superior to a 2MGTR since it could provide a much wider bandwidth and very steep slope when reflective coefficients are suitably chosen.

\section{References}

1. M. Oguma, K. Jinguji, T. Kitoh, T. Shibata, and A. Himeno, "Flatpassband interleave filter with $200 \mathrm{GHz}$ channel spacing based on planar lightwave circuit-type lattice structure," Electron. Lett. 36(15), 1299-1300 (2000).

2. Y. Lai, W. Zhang, and J. A. R. Williams, "All-fibre multichannel flattop filter based on coherent fibre delay line structure," Electron. Lett. 38(10), 473-475 (2002).

3. L. R. Chen, "Design of flat-top bandpass filters based on symmetric phase-shifted long-period fiber gratings," Opt. Commun. 205(5), 271276 (2002).

4. B. B. Dingel and M. Izutsu, "Multifunction optical filter with a Michelson-Gires-Tournois interferometer for wavelength-divisionmultiplexed network system applications," Opt. Lett. 23(11), 10991101 (1998).

5. B. B. Dingel and T. Aruga, "Properties of a novel noncascaded type, easy-to-design, ripple-free optical bandpass filter,' J. Lightwave Technol. 17(8), 1461-1469 (1999).

6. C. Hsieh, R. Wang, Z. J. Wen, I. McMichael, P. Yeh, C. Lee, and W. Cheng, "Flat-top interleavers using two Gires-Tournois etalons as phase-dispersive mirrors in a Michelson interferometer," IEEE Photonics Technol. Lett. 15(2), 242-244 (2003).

7. G. Lenz and C. K. Madsen, "General optical all-pass filter structures for dispersion control in WDM systems," J. Lightwave Technol. 17(7), 1248-1254 (1999).

8. P. Yeh, Optical Waves in Layered Media, p. 150, Wiley, New York (1988). 